\title{
Foreword
}

\section{Ventricular Tachycardia Ablation}

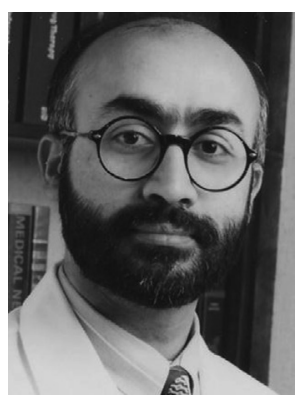

Ranjan K. Thakur, MD, MPH, MBA, FACC, FHRS

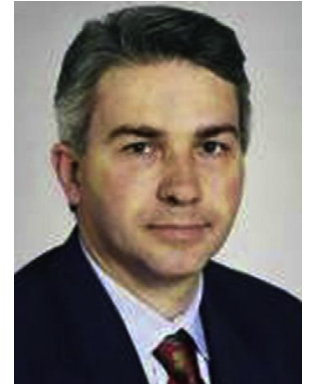

Andrea Natale, MD, FACC, FHRS, FESC

Consulting Editors

Ventricular arrhythmias and sudden cardiac death were the main focus of cardiac electrophysiology in its nascent years. As the cause of ventricular tachycardia (VT), its mechanisms and substrates became better understood, attempts at ablation were undertaken, first by using surgical techniques (endocardial resection \pm cryoablation and ventricular exclusion) and then by using direct current shocks. Other energy sources, such as laser ablation, were also investigated, but later supplanted by radiofrequency (RF) ablation. Now, catheter-based RF ablation is the predominant method for VT ablation used worldwide.

VT ablation has evolved into a field by itself, with a body of literature that's vast, and there are experts who devote a major part of their clinical and research efforts to these arrhythmias.

This issue of Cardiac Electrophysiology Clinics focuses on VT ablation in patients with some form of structural heart disease. The editors have invited experts in the field to shed light on anatomic considerations during VT ablation and electrocardiographic and catheter localization of the VT ablation site using various techniques; each of these localizing techniques are discussed in detail, endocardial and epicardial ablation as well as ablation in various types of heart disease.

We congratulate the editors and contributors for readable and practical reviews relevant to the clinical electrophysiologist. This issue of Cardiac Electrophysiology Clinics should be essential reading for clinicians in practice, because it provides a good review of relevant clinical matters, and for electrophysiology fellows in training, who are developing their foundational knowledge in electrophysiology.

Ranjan K. Thakur, MD, MPH, MBA, FACC, FHRS Sparrow Thoracic and Cardiovascular Institute Michigan State University 1200 East Michigan Avenue, Suite 580 Lansing, MI 48912, USA

Andrea Natale, MD, FACC, FHRS, FESC Texas Cardiac Arrhythmia Institute

Center for Atrial Fibrillation at

St. David's Medical Center 1015 East 32nd Street, Suite 516

Austin, TX 78705, USA

E-mail addresses:

thakur@msu.edu (R.K. Thakur) andrea.natale@stdavids.com (A. Natale) 